\title{
Моделирование распространения сигналов в оптических волоконных линиях связи
}

\author{
С.А. Богданов \\ Новосибирский государственный университет \\ *E-mail: s.bogdanov@g.nsu.ru
}

DOI:10.31868/RFL2018.134

Постоянный рост объемов передачи информации по оптическим каналам много лет обеспечивался ростом скорости передачи данных, путем повышения частоты модуляции. В настоящее время эта частота достигла терагерцового диапазона, где становятся существенными дисперсионные и нелинейные искажения сигнала в оптическом волокне [1]. Для устранения нелинейнодисперсионных искажений сигнала был предложен способ «back propagation», т.e. восстановление исходного сигнала путем численного решения «назад» по линии уравнения, описывающего эволюцию огибающей поля в оптическом волокне. Это уравнение (в скалярном случае) оказалось нелинейным уравнением Шредингера (НУШ) [1], для решения которого существует ряд численных методов, в частности, Split Step Fourier Method (SSFM). Однако SSFM и другие численные методы, основанные на разностной аппроксимации НУШ, оказались недостаточно эффективны для практических приложений. Более эффективный способ решения предлагает метод обратной задачи рассеяния (ОЗР). Для этого метода совсем недавно в институте Автоматики и Электрометрии СО РАН были разработаны быстрые алгоритмы решения прямой и обратной задач рассеяния, основанные на теплицевой симметрии алгебраизованной системы интегральных уравнений Гельфанда - Левитана - Марченко.

В данной работе впервые для задачи передачи информации по оптической линии проводится сравнение численных решений НУШ, полученных методом SSFM и методом, основанным на решении прямой и обратной задач рассеяния, для случая нормальной дисперсии. Исследуются пределы применимости и ограничения ОЗР-метода, возникающие из-за плохой обусловленности обратных задач.

\section{Литература}

[1] G.P. Agrawal, Fiber-Optic Communication Systems, 4th ed. Wiley-Blackwell (2010) 